\title{
Functionalization of UV-curing adhesives for surface-integrated micro-polymer optical fibers
}

\author{
B. Hachicha ${ }^{\mathrm{a}}$ and L. Overmeyer ${ }^{\mathrm{a}}$ \\ ${ }^{a}$ Institute of Transport and Automation Technology, Leibniz Universitaet Hannover, \\ An der Universitaet 2, 30823 Garbsen, Germany
}

\begin{abstract}
Polymer optical waveguides, especially single-mode waveguides are increasingly used for short distance communication, as well as for sensing applications. The realization of a working communication route requires different and sequentially realized steps. Generally, these steps are the packaging of semiconductor beam senders and receivers, the fabrication of an optical waveguide, the preparation of its end-facets, the alignment of different elements along their optical axis and the integration into a desired communication route. The development of a process, which integrates all these steps for planar surfaces, offers a reduction in time and an increase in flexibility. A sub-step toward such a highly automated system is the integration of optical waveguides into the planar surface. In this context, we are investigating the use of the micro-dispensing process to realize this integration step. We functionalize UV-curing adhesives as cladding for micro-optical cores as well as for inherent bonding to the substrate surface.

For this purpose an optical characterization of the adhesives is necessary for an adequate core and cladding material combination. A flow behavior characterization is also relevant in order to analyze the used dispensing process with the selected adhesive. Finally, a mechanical characterization is done to test the adhesion of the core to the adhesive, as well as the adhesive to the substrate surface.

In this paper we present a summary of the realized characterization of the selected polymer. Based on experiment results we infer limits and opportunities of this method.
\end{abstract}

Keywords: optical, communication, surface integrated, waveguide, optic, optronic

\section{INTRODUCTION}

The integration of optical waveguides into different substrate types is an a current issue of scientific research. For optical PCB's, the integration allows a high optical data transfer rate up to Terabit/s. ${ }^{1-3}$ Apart from communication tasks, the sensing properties of optical waveguides ${ }^{4}$ are also used for surface and structure health monitoring allowing a local distributed measurement of temperature and strain changes. ${ }^{5-9}$ Another trend in scientific work is the development of innovative production methods such as flexography ${ }^{10}$ or Inkjet $^{11}$ printing. Hot embossing ${ }^{12}$ of sensors ${ }^{13,14}$ and waveguides is also investigated for an integration in planar polymer foil to realize optical measurement principles. This recent progress in scientific research supports the possibility of an integration of sensing optical waveguides into industrial machines and part surfaces. This integration is advantageous in many perceptions. On the one hand structure health monitoring of machines during processing ${ }^{15}$ supports the possibility of an online-closed loop. On the other hand structure health monitoring of parts helps to accumulate life cycle data. This data can be used for condition oriented maintenance ${ }^{16}$ and use-oriented product design. ${ }^{17}$ Furthermore, using the surface integrated waveguides for communication provides an electromagnetic interference immunity, flexible structures and a high bandwidth.

Innovative methods such as printing or hot-embossing of optical waveguide, ${ }^{10-12,18}$ or the more established methods such as lithography or pattering integrate waveguides into planar systems with a flexible polymer- or semiconductor-based material. ${ }^{19,20}$ However, flexible integration into metallic parts is not yet established. To allow such an integration, we are investigating the use of micro-dispensing process. The idea is based on four steps as presented in fig.1: In the first step a trench structure is realized on the substrate surface along the desired

Further author information: (Send correspondence to B. Hachicha)

B. Hachicha: E-mail: bechir.hachicha@ita.uni-hannover.de, Telephone: 004951176218321

Integrated Optics: Devices, Materials, and Technologies XX, edited by Jean-Emmanuel Broquin, Gualtiero Nunzi Conti, Proc. of SPIE Vol. 9750, 97500J - (c) 2016 SPIE

CCC code: $0277-786 \mathrm{X} / 16 / \$ 18 \cdot$ doi: $10.1117 / 12.2212772$ 

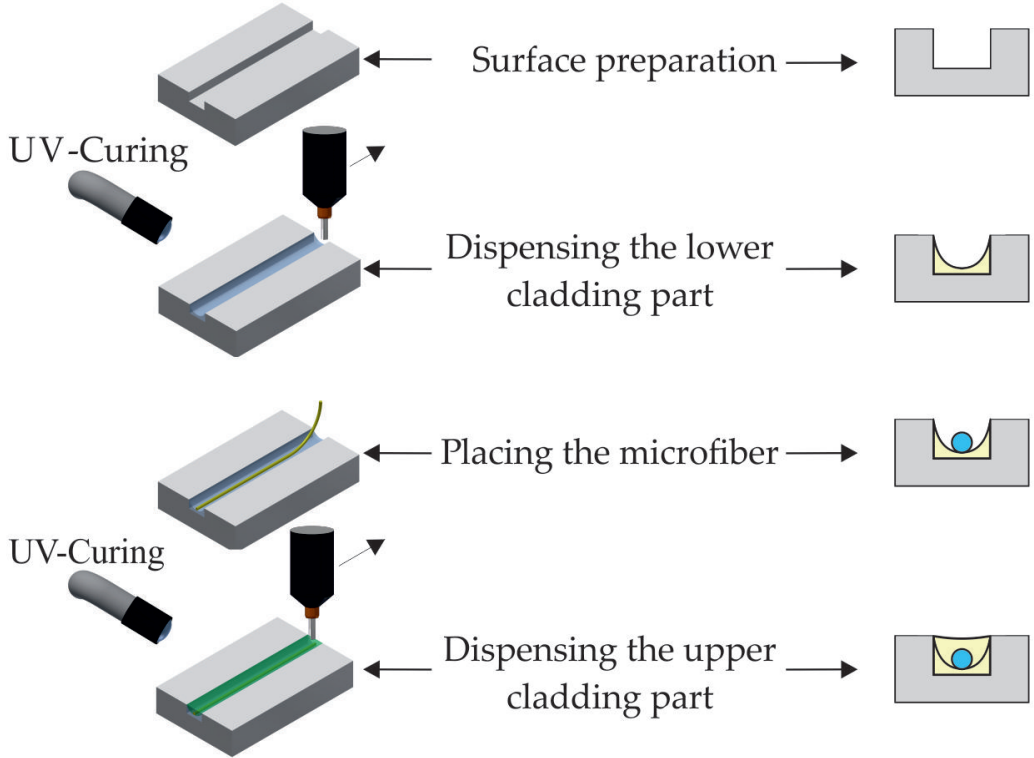

Figure 1. The process steps for a surface integration of micro-polymer fibers

path. The second step consists of dispensing a UV-curing polymer adhesive along the path. This step realizes the lower cladding layer of the waveguide and at the same time the bonding to the substrate surface. In the third step the microfiber core is placed on the first cladding part. The fourth step is realized similar to the first one and the upper cladding layer is dispensed. The dispensing is followed by the curing using UV-radiation. With this method a step-index waveguide is integrated to the surface. The adhesive is functionalized as a cladding material in addition to its bonding function.

The next step is the end-facet preparation for a butt coupling using hot-cleaving and index-matching polymer as presented in a previous paper. ${ }^{21}$ The coupling is realized to sender and receiver, which are also bonded on the substrate surface. In a previous work, ${ }^{22}$ we presented the determined deviation, tilt, pitch and yaw limits using optical simulation for a coupling efficiency $\eta=0.8$. These limits were determined for a certain optical step-index waveguide with a defined geometry and optical properties, such as the numerical aperture $A_{N}$. The stability of these properties is relevant for the coupling efficiency and the validity of the determined limits. A variation in geometry leads to the variation of the core position in the cladding. A variation of optical properties leads to the variation of the numerical aperture $A_{N}$. An overview of the process parameters, which directly affect the geometrical and optical properties, are presented in fig.2. The lower cladding part is characterized with the contact angle $\theta$, the height $h$, as well as the refractive index $n_{\text {clad }}$. The resulting contact angle depends on the surface tension of the used liquid polymer as well as the surface energy of the substrate. The height $h$ depends on the dispensed polymer volume $V$, which in its turn depends on the viscosity of the polymer, the adjusted parameters for the dispensing method such as volume flow $\dot{V}$, as well as the dispensing velocity $v$ along the surface adjusted using the gantry robot. Apart from the trench geometry and surface roughness of the substrate, the height $h$ and the positioning system in the gantry robot define the position $P(x, y)$ of the core. The liquid polymer has a certain refractive index in liquid state, which becomes another value after the curing and cross-linking of the polymer chains. The curing system and its adjustment parameters, such as intensity, curing time or curing distance define the curing conditions and consequently affect the refractive index value in solid state.

We analyzed the first step of the presented integration method (the dispensing of the lower cladding part) and its stability in this work. We want to determine the effect of process parameters deviation on geometrical and optical properties of the lower cladding. We chose the materials and trench geometry summarized in tab.1 for our tests. 


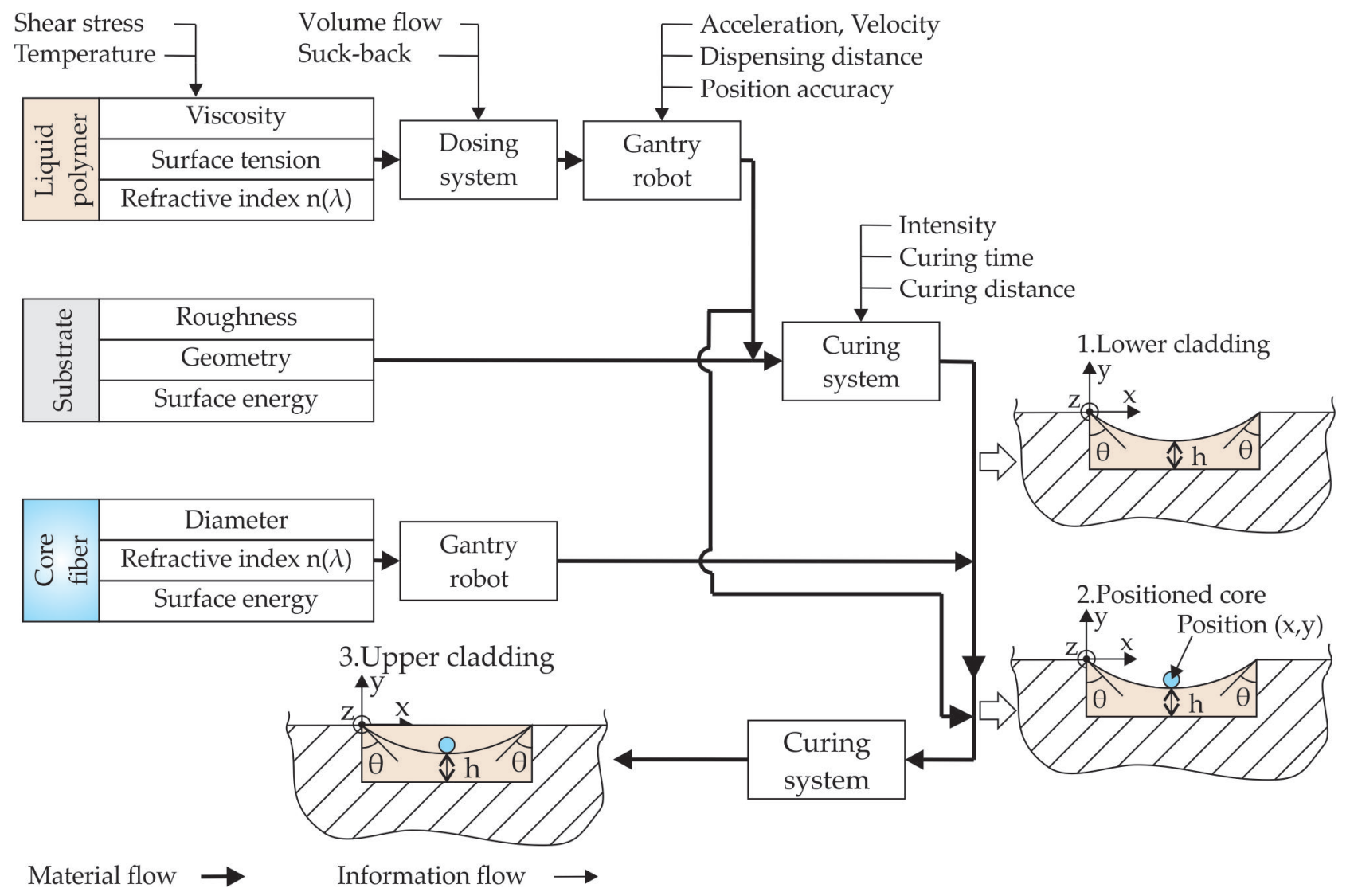

Figure 2. Modell of the micro-POF integration method and its adjustment parameters

\begin{tabular}{|c|c|c|}
\hline \multicolumn{3}{|c|}{ Substrate } \\
\hline Material & Description & Trench geometry \\
\hline Alplan & AI Mg4.5Mn0.7 & $\begin{array}{c}\text { width } 300 \pm 9.7 \mu \mathrm{m} \\
\text { depth } 100 \pm 5.3 \mu \mathrm{m}\end{array}$ \\
\hline \hline \multicolumn{2}{|c|}{ Cladding } \\
\hline Material & Description & $\begin{array}{c}\text { Refractive index } \\
\text { (liquid state, } \lambda=633 \mathrm{~nm} \text { ) }\end{array}$ \\
\hline $\begin{array}{c}\text { UV-curing adhesive } \\
\text { Polytec UV2108 }\end{array}$ & $\begin{array}{c}\text { Methacrylate/ } \\
\text { Acrylate }\end{array}$ & 1.4356 \\
\hline \hline \multicolumn{2}{|c|}{ Core } \\
\hline Material & Description & $\begin{array}{c}\text { Refractive index } \\
\text { (solid state, } \lambda=589 \mathrm{~nm} \text { ) }\end{array}$ \\
\hline PC & Polycarbonate & 1.5805 \\
\hline
\end{tabular}

Table 1. Description and some properties of the used materials

In the next section, we investigated the wetting behavior of the selected liquid polymer to the substrate surface and the reproducibility of the dispensing process. In the third section, we realized an optical characterization of the adhesive in solid state by measuring the transmission and reflexion values according to the variation of the curing parameters. In the fourth section, the adhesion of the cured polymer is considered. In the final section, we conclude with the different considerations to infer aspects for the controlling of the presented method from the realized investigations and their consequences. We also give suggestions for necessary future work to strengthen our conclusions. 


\section{ANALYSIS OF THE DISPENSING PROCESS}

\subsection{Wetting and interfacial dynamic of used adhesive}

The used adhesive as cladding material is dispensed along the trench structure. In the boundary surface between substrate and cladding material an interfacial phenomena is taking place defining the wetting property between these partners. This wetting property can be characterized by the surface tension $\sigma_{S}$ (S: solid) between the substrate and ambient gas, the surface tension $\sigma_{L}$ (L:liquid) between the liquid polymer and ambient gas, the surface tension $\sigma_{L S}$ between the used liquid polymer and substrate, as well as by the contact angle $\theta$ between the different surface tensions as defined by the Young-equation: ${ }^{23}$

$$
\cos \theta=\frac{\sigma_{S}-\sigma_{L S}}{\sigma_{L}} .
$$

If $\sigma^{p}$ the polar component and $\sigma^{d}$ the disperse component of the surface tension of a liquid and a solid are known, the surface tension $\sigma_{L S}$ can be determined by the following equation: ${ }^{24}$

$$
\sigma_{L S}=\sigma_{S}+\sigma_{L}-2 \cdot\left(\sqrt{\sigma_{S}^{d} \cdot \sigma_{L}^{d}}+\sqrt{\sigma_{S}^{p} \cdot \sigma_{L}^{p}}\right) .
$$

Considering the finite volume element $d V$ of the dispensed polymer along the trench, its cross-section $A$ can be characterized by the height $h$ and the contact angle $\theta$ as presented in fig.2. The capillary rise at an infinite vertical board depends on the distance to this board. However, if the considered volume is small enough, the effect of gravity can be neglected and the capillary rise can be considered as circular. In fig. 3 the geometrical relation of the dispensed first cladding part is described based on this approach. The dimension $c$ in this figure represents the sagitta of the presented circle. The height $h$ and the volume $d V_{\max }$ can be formulated as

$$
\begin{aligned}
h & =b-c \\
h & =b-\frac{a}{2} \cdot\left(\frac{1-\sin \theta}{\cos \theta}\right), \\
d V_{\max } & =A_{\max } \cdot d l, \\
d V_{\max } & =\left(a \cdot b-\frac{a^{2}}{8 \cdot \cos \theta^{2}} \cdot((\pi-2 \theta)-\sin (\pi-2 \theta))\right) \cdot d l,
\end{aligned}
$$

where $a$ is the trench width and $b$ the trench depth. We define the minimal volume as the volume where $h=0$. This means that the circular shape of the dispensed cladding material is tangential to the trench bottom. The volume $d V$ should be between $d V_{\min }$ and $d V_{\max }$ :

$$
\begin{aligned}
A_{\min } \cdot d l & <d V \leq A_{\max } \cdot d l \\
\left.\left(a \cdot h-\frac{a^{2}}{8 \cdot \cos \theta^{2}} \cdot((\pi-2 \theta)-\sin (\pi-2 \theta))\right)\right) d l<d V & \leq\left(a \cdot b-\frac{a^{2}}{8 \cdot \cos \theta^{2}} \cdot((\pi-2 \theta)-\sin (\pi-2 \theta))\right) d l, \\
0 & <\quad h \quad \leq b-\frac{a}{2} \cdot\left(\frac{1-\sin \theta}{\cos \theta}\right) .
\end{aligned}
$$




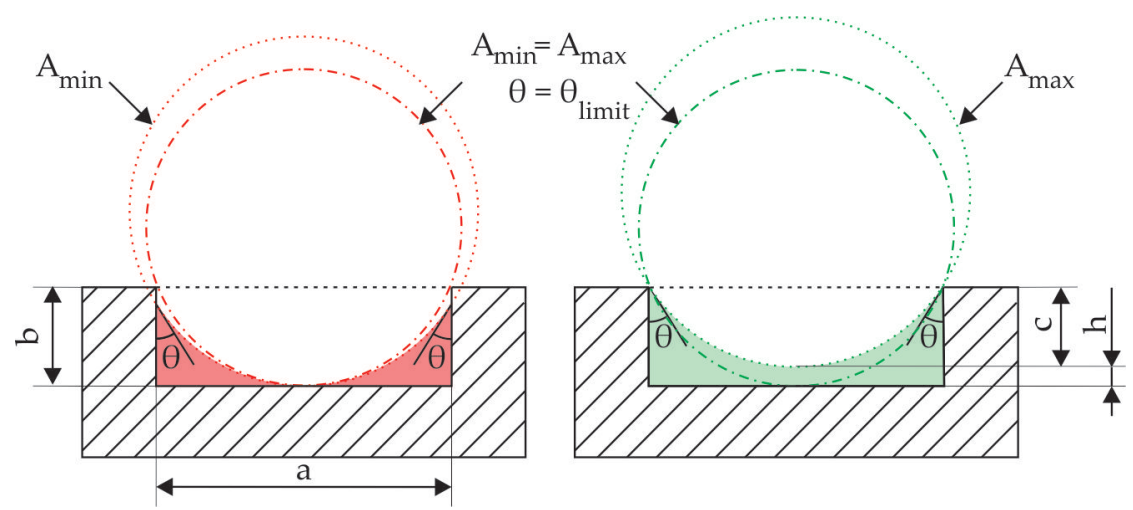

Figure 3. Geometrical description of the lower cladding part cross-section

To determine these value limits we conducted a surface tension measurement of the liquid polymer using the pendant drop method according to DIN 55660-3 and the substrate surface energy by measuring the contact angle according to DIN 55660-2. The results are summarized in tab.2.

\begin{tabular}{|c||c||cc|}
\hline Substarte Material: & Surface Energy & disperse component & polar component \\
& $\sigma$ & $\sigma^{d}$ & $\sigma^{p}$ \\
$\mathrm{Al} \mathrm{Mg} 4.5 \mathrm{Mn} 0.7$ & $35.13 \pm 4.9 \mathrm{mN} / \mathrm{m}$ & $28.13 \pm 11.35 \mathrm{mN} / \mathrm{m}$ & $7 \pm 1.83 \mathrm{mN} / \mathrm{m}$ \\
\hline \hline UV-Curing adhesive: & Surface tension & disperse component & polar component \\
& $\sigma$ & $\sigma^{d}$ & $\sigma^{p}$ \\
Polytec UV2108 & $37.61 \pm 0.66 \mathrm{mN} / \mathrm{m}$ & $36.63 \pm 0.63 \mathrm{mN} / \mathrm{m}$ & $0.98 \pm 0.04 \mathrm{mN} / \mathrm{m}$ \\
Methacrylate/Acrylate & &
\end{tabular}

Table 2. Measured surface tension $\sigma_{L}$ and $\sigma_{S}$

Based on these values the expected contact angle $\theta=32.19$ between substrate and liquid polymer can be theoretically determined by using equations 1 and 2 . This value is between 0 and $\pi / 2$, which represents a partial wetting case. ${ }^{25}$ To validate this value we realized several measurements of the contact angle based on DIN 5560-2. The measured values are time-dependent and start on an average of $31.64 \pm 1.17^{\circ}$ till reaching a quasi-stationary value of $22.42 \pm 0.04^{\circ}$ as presented in fig.4.

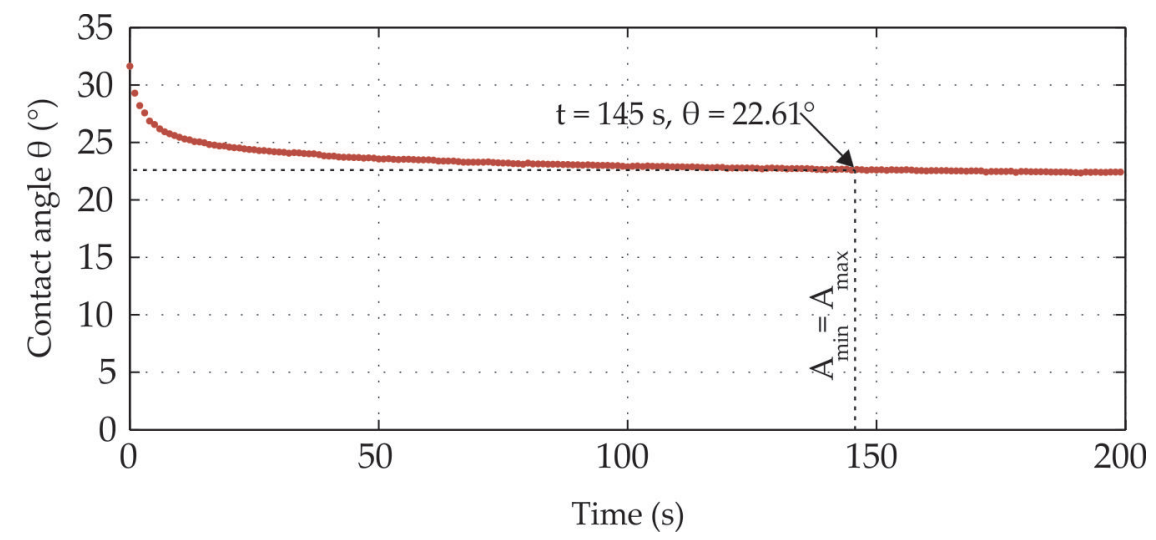

Figure 4. Time dependence of the contact angle between the adhesive and the used substrate

Considering the trench width $a=300 \mu \mathrm{m}$ and depth $b=100 \mu \mathrm{m}$, the contact angle from $t=0$ to $t=200 \mathrm{~s}$, the maximal and minimal cross-section surface $A$ can be calculated using eq.7. From this equation, we can 
also conclude that $A_{\max }=A_{\min }$ for the contact angle $\theta_{\text {limit }}=22.61^{\circ}$. This case is presented in fig. 3 with the dot-dash line. Contact angles smaller than $\theta_{\text {lim }}$ mean that the trench bottom will not be completely coated and the capillary rise will be higher than the trench edge. In this case the dispensed polymer will not be flush to the substrate surface. In fig.4 the limit value $\theta_{\text {limit }}$ is marked and the value is reached by $t \approx 145 \mathrm{~s}$. In the following subsection, we will consider a curing during the first 5 seconds, which means a contact angle value between ca. $26^{\circ}$ and $32^{\circ}$. Based on the maximal value $\theta=32^{\circ}$, a characterization of the dispensing method is realized to test its influence on the dispensing volume and so the height of the first cladding, whose deviation is relevant for the core position.

\subsection{Reproducibility of the dispensing process}

The most common dispensing methods use time-pressure, piston, endless-screw, peristatic-valve, Ink-Jet or jetvalve techniques. ${ }^{26}$ In our case we want to assure a volumetric stable line dispensing, which is best provided by the endless-screw method using conventional dispensing tips. ${ }^{26}$ For this purpose we used the dosing unit eco-pen 450 from ViscoTec, which is a precision-volume dosing unit based on the endless-screw technique. Its control device allows an adjustment of the volume flow $\dot{V}$ from 0.5 to $6 \mathrm{ml} / \mathrm{min}$.

In the presented integration method, the total volume of the lower cladding part depends on the length of the trench. By defining a certain volume flow, the necessary time $t$ to dispense the desired volume can be calculated. The dosing unit is fixed at a gantry robot, whose forward velocity $v$ is adjusted based on the trench length and the necessary dispensing time $t$. The minimal and maximal velocity at a defined volume flow can be determined using eq. 7 by:

$$
\begin{aligned}
d V_{\min }<\dot{V} \cdot t & \leq d V_{\max } \\
\left(a \cdot h-\frac{a^{2}}{8 \cdot \cos \theta^{2}} \cdot((\pi-2 \theta)-\sin (\pi-2 \theta))\right) d l<\dot{V} \cdot t & \leq\left(a \cdot b-\frac{a^{2}}{8 \cdot \cos \theta^{2}} \cdot((\pi-2 \theta)-\sin (\pi-2 \theta))\right) d l, \\
\left(a \cdot h-\frac{a^{2}}{8 \cdot \cos \theta^{2}} \cdot((\pi-2 \theta)-\sin (\pi-2 \theta))\right) d l<\dot{V} \cdot \frac{d l}{v} & \leq\left(a \cdot b-\frac{a^{2}}{8 \cdot \cos \theta^{2}} \cdot((\pi-2 \theta)-\sin (\pi-2 \theta))\right) d l, \\
\frac{\dot{V}}{\left(a \cdot b-\frac{a^{2}}{8 \cdot \cos \theta^{2}} \cdot((\pi-2 \theta)-\sin (\pi-2 \theta))\right)}<\quad v & \leq \frac{\dot{V}}{\left(a \cdot h-\frac{a^{2}}{8 \cdot \cos \theta^{2}} \cdot((\pi-2 \theta)-\sin (\pi-2 \theta))\right)}
\end{aligned}
$$

In fig. 5 this relation is graphically presented, as well as the process limits. These limits are defined by the volume flow and velocity adjustment range from respectively 0.04 to $6 \mathrm{ml} / \mathrm{min}$ and 0 to $3.8 \mathrm{~m} / \mathrm{s}$. The gantry robot also has a maximal acceleration $a=50 \mathrm{~mm} / \mathrm{s}^{2}$, which means that for the time $t_{\text {delay }}=v_{\min } / a$ and the length $l_{\text {delay }}=a / 2 \cdot t_{\text {delay }}^{2}$ the necessary conditions are not complied.

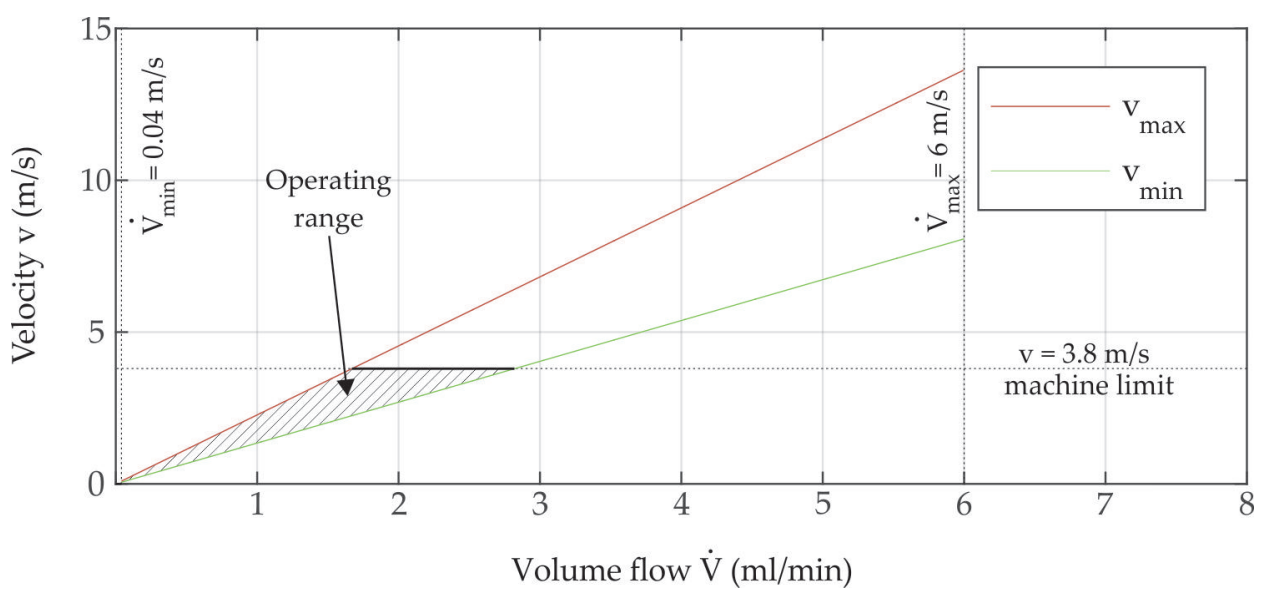

Figure 5. Dispensing velocity in relation to adjusted dispensing volume flow

We realized 3 experiments with 50 measurements each. For a defined volume flow $\dot{V}=0.5 \mathrm{ml} / \mathrm{min}$, we dispensed 3 different lengths $(0.16 \mathrm{~m}, 0,32 \mathrm{~m}, 3,22 \mathrm{~m})$, which corresponds to 3 different dispensing time durations 
$(0.24 \mathrm{~s}, 0.48 \mathrm{~s}, 4.8 \mathrm{~s})$. For each length we repeated the experiment 50 times and weighted the dispensed polymer. Then we determined the total volume flow deviation with the measured weight. The average and the standard deviation are also presented in fig.6. We can conclude that deviations are higher for shorter dispensing times. If we use the velocity limits $v_{\min }$ and $v_{\max }$, the deviation in the volume flow can leads to an off-limit for the system as presented with the hatching surface in fig.6. A volume flow deviation under and over the adjusted value leads to an underfilling by $v_{\max }$ and an overfilling by $v_{\min }$. If the mean velocity $v_{\text {mean }}=\left(v_{\max }+v_{\min }\right) / 2$ is used, the volume flow deviations still fit the process limits. However, these volume flow deviations lead to a $\pm 1.9 \mu \mathrm{m}, \pm 2.1 \mu \mathrm{m}$ and $\pm 4.7 \mu \mathrm{m}$ deviation from the height $h$ for respectively $4.8 \mathrm{~s}, 0.48 \mathrm{~s}$ and $0.24 \mathrm{~s}$. This shows a deviation from the core position $\mathrm{P}(\mathrm{x}, \mathrm{y})$. This deviation affects the coupling efficiency, since the real position $P$ does not correspond to the required position. An active regulation of this deviation by changing the velocity based on eq.9 is possible in order to fit into the process limits. For this purpose we need to measure the variation in the volume flow during the process. This cannot be realized directly, but indirectly using a fluid pressure sensor. In this case, we used the fluid sensor Flowplus16 from ViscoTec and measured the flow pressure on-line while dispensing. In fig.6, an example of the pressure profile for the 3 dispensing durations is presented. The pressure increases during the dispensing time and reaches the maximum at the end of it. The values of $0.5 \mathrm{bar}$ and $3.3 \mathrm{bar}$ are reached respectively at $0.48 s$ and $4.8 \mathrm{~s}$. The pressure begins to decrease immediately by the end of the dispensing time. In the case of the short dispensing time of $0.24 \mathrm{~s}$, the pressure value does not change. This means that the dispensing unit is not adequate for a short distance in range of $100 \mathrm{~mm}$. These measurements show a correlation between dispensing time and pressure, and consequently between pressure values and the volume flow deviation. We calculated a Spearman's rank correlation coefficient of ca. 0.5 between the volume flow deviations and the maximal reached pressure values. This proves that a correlation function can be formulated between the flow pressure and the volume flow, which can be used to regulate the system on-line. To determine a correlation function with a minimum error coefficient, more precise measurements are planed dispensing directly on a time-recording anlytical balance. Through this it is possible to directly compare the pressure and the volume flow behavior through the duration.

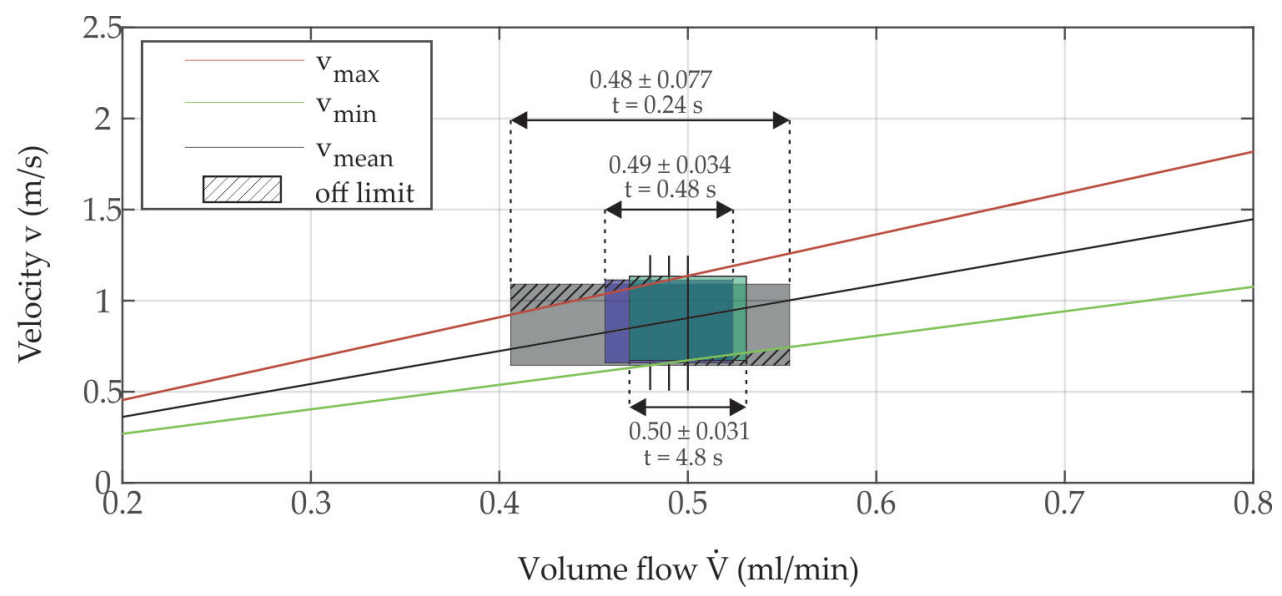

Figure 6. Volume flow deviations for different dispensing times

After dispensing the lower cladding part, curing takes place. This process step also has several adjustment parameters such as the beam intensity, the distance between the UV-beam and polymer, the size of the UV-beam spot, as well as the curing duration. In the next section possible impacts of changing these parameters on optical properties are investigated.

\section{OPTICAL CHARACTERIZATION OF CURED CLADDING MATERIAL}

The selected adhesive polytec UV2108 used as cladding material should have a refractive index lower than the index of refraction of core material $\left(\mathrm{PC}, n_{\text {core }}=1.5805\right.$ at $633 \mathrm{~nm}$ ) to achieve the step-index waveguide. The data-sheet of the selected adhesive recommends a curing with a spectral output between 320 and $500 \mathrm{~nm}$ during 


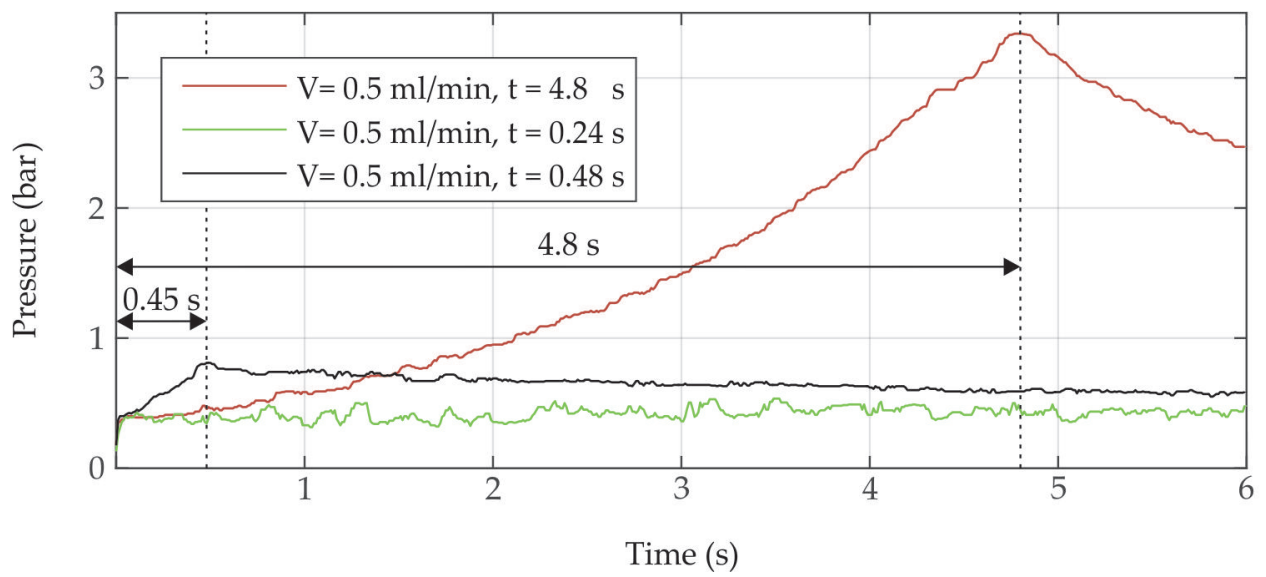

Figure 7. Pressure profile for different dispensing durations

$t_{r}=5 \mathrm{~s}$ by a $d_{r}=0.05 \mathrm{~mm}$ thickness. The recommended intensity $I_{r}$ should be adjusted between $I_{r_{\min }}=0.6$ and $I_{r_{\max }}=1 \mathrm{~mW} / \mathrm{mm}^{2}$. From these data sheet recommendations, it is possible to interpolate an approach for the necessary Intensity $I$ depending on the polymer thickness $d$ and the curing duration $t$ as follows:

$$
I(d, t)=\frac{I_{r} \cdot t_{r}}{d_{r}} \cdot \frac{d}{t} .
$$

After the curing based on this recommendations we measured the refractive index $n_{\text {cladding }}=1.51$ at $\lambda=590 \mathrm{~nm}$ using a profilometer from Rink Electronic.

We designed an experiment to determine the influence of curing parameters on optical properties. We varied these parameters based on eq.10 as presented in tab.3 and realized two samples for each variation. Subsequently, we realized 6 transmission and reflexion measurements at each sample using the spectrophotometry instrument Perkin Elmer Lambda 950 at a wavelength between 200 and $2000 \mathrm{~nm}$. From the reflection and transmission values, we can recognize if there is a deviation due to the variation of curing parameters. Based on this deviation, we can also conclude the existence of a refractive index deviation through the Fresnel equation.

\begin{tabular}{|c|c|c|c|c|c|c|c|}
\hline \# & Dimension & $\begin{array}{c}\text { Thickness } \\
\text { d }\end{array}$ & $\begin{array}{c}\text { Distance } \\
\mathrm{a}\end{array}$ & & $\begin{array}{c}\text { Intensity } \\
\text { I }\end{array}$ & $\begin{array}{c}\text { Duration } \\
t\end{array}$ & Comment \\
\hline 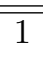 & $\overline{e 1 "}$ & $0.6 \pm 0.09 \mathrm{~mm}$ & $20 \mathrm{~mm}$ & $\overline{0.72}$ & $=0.02 \mathrm{~W} / \mathrm{cm}^{2}$ & $\overline{5 \mathrm{~s}}$ & 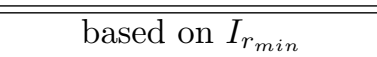 \\
\hline 2 & $\oslash 1 "$ & $0.6 \pm 0.09 \mathrm{~mm}$ & $10 \mathrm{~mm}$ & 0.72 & $\pm 0.02 \mathrm{~W} / \mathrm{cm}^{2}$ & $5 \mathrm{~s}$ & based on $I_{r_{\min }}$ \\
\hline 3 & $\oslash 1 "$ & $0.6 \pm 0.09 \mathrm{~mm}$ & $10 \mathrm{~mm}$ & 1.2 & $\pm 0.02 \mathrm{~W} / \mathrm{cm}^{2}$ & $5 \mathrm{~s}$ & based on $I_{r_{n}}$ \\
\hline 4 & $\oslash 1 "$ & $0.6 \pm 0.09 \mathrm{~mm}$ & $10 \mathrm{~mm}$ & 4 & $\pm 0.02 \mathrm{~W} / \mathrm{cm}^{2}$ & $1.5 \mathrm{~s}$ & based on $I_{r_{\max }}$ \\
\hline 5 & $\oslash 1 "$ & $0.6 \pm 0.09 \mathrm{~mm}$ & $10 \mathrm{~mm}$ & 4 & $\pm 0.02 \mathrm{~W} / \mathrm{cm}^{2}$ & $0.9 \mathrm{~s}$ & reduced curing duration \\
\hline 6 & $\oslash 1 "$ & $0.6 \pm 0.09 \mathrm{~mm}$ & $10 \mathrm{~mm}$ & 11.9 & $\pm 0.02 \mathrm{~W} / \mathrm{cm}^{2}$ & $0.9 \mathrm{~s}$ & $\begin{array}{l}\text { reduced curing duration } \\
\text { and increased intensity }\end{array}$ \\
\hline
\end{tabular}

Table 3. Used UV-curing parameters for the designed experiments

We used a the Panasonic UV-control device UJ35 with the lamp ANUJ6184 and a $3 \mathrm{~mm}$ spot diameter by a $10 \mathrm{~mm}$ distance. The UV-control device has an UV-intensity sensor, which allows the adjustment of the desired intensity up to $11.9 \mathrm{~W} / \mathrm{cm}^{2}$.

It is difficult to study the effect of changing curing parameters of the whole measured spectrum. For this reason, we picked up 6 relevant wavelengths going from ultra-violet to infra-red and used them for the evaluation of curing parameter effects. The significance of these effects are evaluated based on statistical confidence interval calculation ${ }^{27}$ and the results are summarized in tab.4. We can see that the use of the minimal or maximal required curing intensity does not have any significant effect on transmission or reflexion, as well as a $40 \%$ reduction of the required curing time $t_{r}$ by the maximum required Intensity $I_{r_{\max }}$. However, using a significantly higher 


\begin{tabular}{|c|c|c|c|c|c|c|c|c|c|c|c|c|c|}
\hline \multirow{3}{*}{$\begin{array}{l}\text { Effect on } \\
\text { transmission of }\end{array}$} & \multirow{3}{*}{ Exp. } & \multicolumn{12}{|c|}{$\lambda(n m)$} \\
\hline & & \multicolumn{2}{|c|}{380} & \multicolumn{2}{|c|}{470} & \multicolumn{2}{|c|}{568} & \multicolumn{2}{|c|}{650} & \multicolumn{2}{|c|}{980} & \multicolumn{2}{|c|}{1550} \\
\hline & & Sig & $\%$ & Sig & $\%$ & Sig & $\%$ & Sig & $\%$ & Sig & $\%$ & Sig & $\%$ \\
\hline a: $20 \mathrm{~mm} \rightarrow 10 \mathrm{~mm}$ & $1 \& 2$ & $* * *$ & $\downarrow 11.9$ & $*$ & 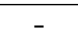 & $*$ & - & $*$ & - & $*$ & - & $*$ & - \\
\hline $\begin{array}{l}I_{\min } \rightarrow I_{\max } \\
\quad \text { curing: }\end{array}$ & $2 \& 3$ & 0 & - & 0 & - & 0 & - & 0 & - & 0 & - & 0 & - \\
\hline 1 step $\rightarrow$ many steps & $3 \& 4$ & * & - & $* *$ & $\uparrow 9.8$ & $* *$ & $\uparrow 10.3$ & $*$ & - & $* *$ & $\uparrow 6.8$ & $* *$ & $\uparrow 7.3$ \\
\hline$t>t_{r}$ & 2 & - & - & * & - & $*$ & - & $*$ & - & $*$ & - & $*$ & - \\
\hline$t<t_{r}$ & $4 \& 5$ & * & & 0 & - & 0 & - & 0 & - & 0 & - & 0 & - \\
\hline$I>I_{r}$ & $\& 6$ & $* * *$ & $\uparrow 35.2$ & 0 & - & 0 & - & 0 & - & 0 & - & $*$ & - \\
\hline \multirow{3}{*}{$\begin{array}{l}\text { Effect on } \\
\text { reflexion of }\end{array}$} & \multirow{3}{*}{ Exp. } & \multicolumn{12}{|c|}{$\lambda(n m)$} \\
\hline & & \multicolumn{2}{|c|}{380} & \multicolumn{2}{|c|}{470} & \multicolumn{2}{|c|}{568} & \multicolumn{2}{|c|}{650} & \multicolumn{2}{|c|}{980} & \multicolumn{2}{|c|}{1550} \\
\hline & & Sig & $\%$ & Sig & $\%$ & Sig & $\%$ & Sig & $\%$ & Sig & $\%$ & Sig & $\%$ \\
\hline a: $20 \mathrm{~mm} \rightarrow 10 \mathrm{~mm}$ & $1 \& 2$ & $* * *$ & $\downarrow 2.2$ & * & - & $*$ & - & $*$ & - & $*$ & - & $*$ & - \\
\hline $\begin{array}{l}I_{\min } \rightarrow I_{\max } \\
\quad \text { curing: }\end{array}$ & $2 \& 3$ & 0 & - & 0 & - & 0 & - & 0 & - & 0 & - & 0 & - \\
\hline 1 step $\rightarrow$ many steps & $3 \& 4$ & 0 & - & $* *$ & $\uparrow 2.4$ & $* *$ & $\uparrow 2.4$ & $* *$ & $\uparrow 2.4$ & $* *$ & $\uparrow 2.4$ & * & - \\
\hline$t>t_{r}$ & $4 \& 2$ & * & - & $* *$ & $\uparrow 2.5$ & $* *$ & $\uparrow 2.5$ & $* *$ & $\uparrow 2.5$ & * & - & $*$ & - \\
\hline$t<t_{r}$ & $4 \& 5$ & 0 & - & 0 & - & 0 & - & 0 & - & 0 & - & 0 & - \\
\hline$I>I_{r}$ & $5 \& 6$ & $* * *$ & $\uparrow 2.3$ & $* * *$ & $\uparrow 2.2$ & $* * *$ & $\uparrow 2.2$ & $* * *$ & $\uparrow 2.2$ & $* * *$ & $\uparrow 2.4$ & $* * *$ & $\uparrow 2.3$ \\
\hline
\end{tabular}

Table 4. Significance (Sig.) of the different effects for the designed experiment.(0): no sig., $\left(^{*}\right)$ : low sig./more experiments are required, $(* *)$ : sig. is available, $(* * *)$ : high sig.

intensity than the maximal required curing intensity leads to an increase of reflexion values for all wavelengths and of the transmission value only in the lower UV-range. Curing the polymer in one step in the required time period $t_{r}$, or curing it in a repetitive loop by dividing the required time in small intervals has almost a significant effect on the transmission and reflexion in the visible spectrum. We also recognized that a longer curing time than $t_{r}$ or a higher intensity than $I_{r}$ increase the reflexion values for the different wavelengths. According to the transmission value, only an increase by $\lambda=380 \mathrm{~nm}$ is to be mentioned for a higher intensity than $I_{r}$.

We can conclude that the control of the UV-beam intensity and the exposure duration to the beam source are important for repeatable optical properties of the used adhesive. The decision if the curing should take place in one step or many has to be consistently applied, since a variation leads to variation in the optical properties.

\section{ADHESION TEST}

Apart from its task as cladding, the used adhesive should realize the bonding between the core and cladding material as well as between the cladding and substrate surface. For this reason, we realized an adhesion test based on DIN EN ISO 2409. In this test we coated the substrate material as well as a polycarbonate substrate (same as core material) with the selected adhesive. The test is performed using a cross-cutting tool, which produces a lattice structure at the coated surface. The lattice structure should be deeper than the bonding surface to scrape the substrate surface. Afterwards generated microscopic images of the lattice structure are compared to the reference table in the norm. 


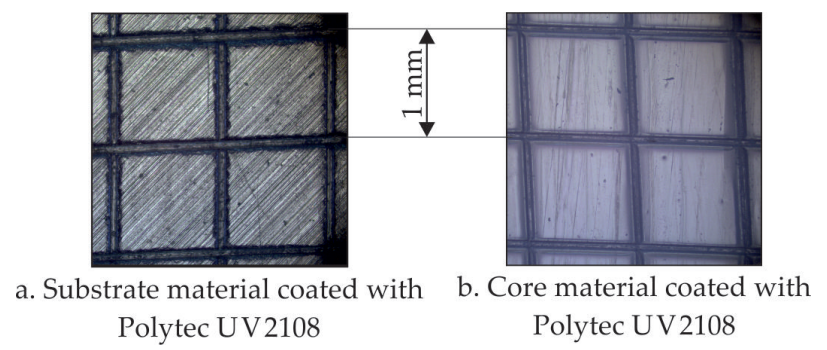

Figure 8. Microscopic image of the realized lattice structure on the coated substrates

The adhesion is considered as optimal, if we get smooth cutting edges and the lattice surfaces do not show a mechanical spalling. Fig.8 shows the obtained results, which fulfill the required conditions for an optimal adhesion.

\section{CONCLUSION AND NEXT STEPS}

In the beginning of this article, we presented our pursued method for a metallic surface integration of micropolymer fibers. Our goal is an automated and robust process with a high repetition accuracy. The weak point in this process is the coupling efficiency with the sender and receiver on the surface. For this purpose, we defined the position deviation, tilt, pitch and yaw limits for a coupling efficiency $\eta=0.8$ in previous work. ${ }^{22}$ These limits were used for designing the necessary actuators to achieve the required positioning accuracy of the core fiber relatively to the sender or receiver position. Furthermore, the efficiency also depends on the coupling method and the end-facet preparation of the integrated waveguide. We analyzed this question in a prior paper ${ }^{21}$ and chose to use hot-cleaving in combination with an index matching polymer as an end-facet preparation method. Another important factor for the coupling efficiency is the repeatability of the integration process and the resulted optical properties of the surface integrated waveguide. For this purpose, we consecrated this article to analyze the dispensing of the first cladding part, which consists of the first step of the presented method. The first cladding part defines the height of the core fiber inside the cladding. Moreover, the cladding refractive index in combination with the core refractive index define the numerical aperture $A_{N}$ of the waveguide. The position of the core in the cladding as well as the numerical aperture are relevant parameters to determine the achievable coupling efficiency and any change leads to a change of the boundary conditions. We tested the influence of process parameters deviation on geometrical and optical properties of the cladding material.

In section 2, we studied the wetting behavior of the used materials and theoretically determined the required volume flow and dispensing velocity to achieve a defined height $h$. Then we analyzed the used dispensing method and stated a time dependent deviation of the volume flow. This deviation has to be counteracted to avoid a resulting height deviation. For this purpose, a monitoring of the volume flow during the dispensing process is necessary. However, this is not directly possible but indirectly using a fluid pressure sensor. A correlation between the volume flow and the measured pressure values was observed. We are planning a further analysis of the volume flow behavior through time in order to determine the correlation function of the pressure values, which will allow the on-line regulation of the dispensing process. Moreover, a further analysis of the dynamic wetting behavior is intended by measuring the dynamic contact angle based on DIN 55660-6. This will complete the time-dependence analysis of the contact angle presented in fig.4.

In section 3, we optically characterized the cladding part by measuring the relative transmission and reflexion values. For this purpose, we cured the adhesive using different parameters and mentioned that especially a higher intensity and higher exposure duration lead to a variation of the optical properties. The curing parameters have to be controlled during the process to ensure a stability of the results. In further works, we are also preparing a series of experiments to directly measure the refractive index using ellipsometry. Furthermore, we will investigate the stability of these results under laboratory ageing conditions, since the waveguide is exposed to UV-radiation, humidity and stress while operating. The adhesion dependence will also be investigated in this context. 

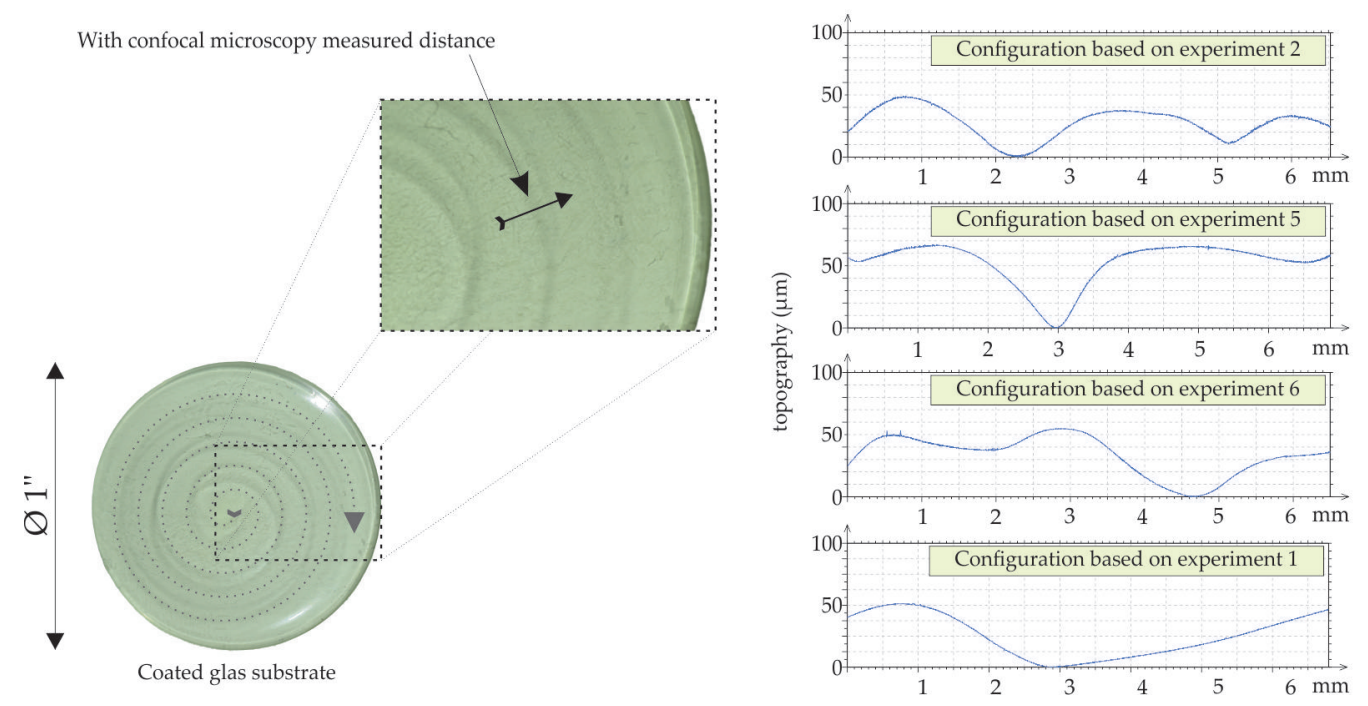

Figure 9. Topography of cured adhesive for different curing paramters

Another important question is the influence of curing parameters and ageing conditions on the final geometry of the cured polymer. In preliminary investigations, we coated 1" glass substrate and cured it in a move involute to a circle under different curing conditions based on exp. 1,2,5 \& 6 from tab.3. Fig.9 shows the different resulting topographies. It is obvious, that the curing conditions affect the final geometry. It remains to determine the correlation with the intensity distribution of the used beam and its relevance for thin structures, such as the dispensed cladding part in our case.

\section{ACKNOWLEDGMENTS}

This research project is part of the Collaborative Research Centre program SFB 653 - Gentelligent Components in their Lifecycle - Utilization of Inherent Information in Production Engineering (www.sfb653.unihannover. de). The authors want to thank the German Research Foundation (DFG) for supporting and funding this research project.

\section{REFERENCES}

[1] Lehmacher, S. and Neyer, A., "Integration of polymer optical waveguides into printed circuit boards," Electronics Letters 36(12), 1052 (2000).

[2] Schow, C., Doany, F., and Kash, J., "Get on the optical bus," IEEE Spectrum 47(9), 32-56 (2010).

[3] Doany, F. E., Schow, C. L., Lee, B. G., A. Budd, R., W. Baks, C., K. Tsang, C., Knickerbocker, J. U., Dangel, R., Chan, B., Lin, H., Carver, C., Huang, J., Berry, J., Bajkowski, D., Libsch, F., and Kash, J. A., "Terabit/s-class optical pcb links incorporating 360-gb/s bidirectional $850 \mathrm{~nm}$ parallel optical transceivers," Journal of Lightwave Technology 30(4), 560-571 (2012).

[4] Pruneri, V., Riziotis, C., Smith, Peter G. R., and Vasilakos, A., "Fiber and integrated waveguide-based optical sensors," Journal of Sensors 2009, 1-3 (2009).

[5] Rosalie, C., Norman, P., Smithard, J., Davis, C., and Rajic, N., "Optical fibre sensing of high order lamb waves for structural health monitoring," in [ASME 2012 Conference on Smart Materials, Adaptive Structures and Intelligent Systems], 723 (Wednesday 19 September 2012).

[6] Chang, X., He, X., Hu, J., and Li, J., "Experimental research on embedded fiber bragg grating sensors network for solid rocket motors health monitor," in [2008 First International Conference on Intelligent Networks and Intelligent Systems (ICINIS)], 170-173.

[7] Ramakrishnan, M., Rajan, G., Semenova, Y., and Farrell, G., "Hybrid fiber optic sensor system for measuring the strain, temperature, and thermal strain of composite materials," IEEE Sensors Journal 14(8), 2571-2578 (2014). 
[8] Wu, M.-C., Winfree, W. P., Moore, J. P., Peters, K. J., Ecke, W., and Matikas, T. E., "Fiber optic thermal health monitoring of composites," in [SPIE Smart Structures and Materials + Nondestructive Evaluation and Health Monitoring], SPIE Proceedings, 764808-764808-9, SPIE (2010).

[9] Wild, G., Allwood, G., and Hinckley, S., "Distributed sensing, communications, and power in optical fibre smart sensor networks for structural health monitoring," in [2010 Sixth International Conference on Intelligent Sensors, Sensor Networks and Information Processing (ISSNIP)], 139-144.

[10] Wolfer, T., Bollgruen, P., Mager, D., Overmeyer, L., and Korvink, J. G., "Flexographic and inkjet printing of polymer optical waveguides for fully integrated sensor systems," Procedia Technology 15, 522-530 (2014).

[11] Loffelmann, U., Mager, D., and Korvink, J. G., "Conductive and transparent gel microstructures fabricated by inkjet printing of ionic liquid based fluids," in [2012 7th IEEE International Conference on Nano/Micro Engineered and Molecular Systems (NEMS)], 312-317.

[12] Rezem, M., Günther, A., Rahlves, M., Roth, B., and Reithmeier, E., "Hot embossing of polymer optical waveguides for sensing applications," Procedia Technology 15, 514-521 (2014).

[13] Xiao, Y., Pichler, E., Hofmann, M., Bethmann, K., Köhring, M., Willer, U., and Zappe, H., "Towards integrated resonant and interferometric sensors in polymer films," Procedia Technology 15, 692-702 (2014).

[14] Kelb, C., Reithmeier, E., and Roth, B., "Foil-integrated 2d optical strain sensors," Procedia Technology 15, 711-716 (2014).

[15] Denkena, B., Litwinski, K. M., and Boujnah, H., "Process monitoring with a force sensitive axis-slide for machine tools," Procedia Technology 15, 416-423 (2014).

[16] M. Winkens, M. Goerke, P. Nyhuis, "Use of life cycle data for condition-oriented maintenance," International Journal of Social, Education, Economics and Management Engineering (9), 1184-1187 (2015).

[17] Neubach, M., [Wissensbasierte Rückführung von Produktnutzungsinformationen in die Produktentwicklung im Rahmen einer Product-Lifecycle-Management-(PLM)-Lösung], vol. Bd. 2010,2 of Maschinenbauinformatik, Shaker, Aachen (2010).

[18] Sagmeister, M., Lamprecht, B., Kraker, E., Köstler, S., Ribitsch, V., Galler, N., Ditlbacher, H., Krenn, J. R., Ungerböck, B., Abel, T., and Mayr, T., "Screen-printed polymer waveguides for integrated optics," Applied Physics B 111(4), 647-650 (2013).

[19] Joseph Carl Dingeldein, [Direct Write Fabrication of Waveguides and Direct Write Fabrication of Waveguides and Interconnects for Optical Printed Wiring Boards].

[20] Yin, D., Deamer, D. W., Schmidt, H., Barber, J. P., and Hawkins, A. R., "Integrated optical waveguides with liquid cores," Applied Physics Letters 85(16), 3477 (2004).

[21] Hachicha B., O. L., "Metal surface inherent micro-pofs," in [24th International Conference on POFs], 295-301.

[22] Bechir Hachicha, M., Kuklik, J., and Overmeyer, L., "Simulation of coupling efficiency for surface integration of optical waveguides," in [2015 6th International Conference on Modeling, Simulation, and Applied Optimization (ICMSAO)], 1-7.

[23] Miller, C. A. and Neogi, P., eds., [Interfacial phenomena: Equilibrium and dynamic effects], vol. 139 of Surfactant science series, CRC/Taylor \& Francis, Boca Raton, Fla., 2. ed. ed. (2008).

[24] Kaelble, D. H., "Dispersion-polar surface tension properties of organic solids," The Journal of Adhesion 2(2), 66-81 (1970).

[25] Starov, V. M., Radke, C. J., and Velarde, M. G., [Wetting and spreading dynamics], vol. v. 138 of Surfactant science series, CRC Press/ Taylor \& Francis, Boca Raton (2007).

[26] Fahlbusch, T., [Dispensieren polymerer Lichtwellenleiter: Univ., Diss.-Hannover, 2007], vol. 2007,1 of Berichte aus dem ITA, PZH Produktionstechn. Zentrum, Garbsen (2007).

[27] Kleppmann, W., [Versuchsplanung: Produkte und Prozesse optimieren], Praxisreihe Qualitätswissen, Hanser, München, 8., überarb. aufl. ed. (2013). 